\title{
Impacte do Aumento das Taxas Moderadoras na Procura dos Cuidados de Saúde Primários na USF do Parque
}

António Ramos, ${ }^{1}$ Catarina Rúbio, ${ }^{2}$ Diogo Rodrigues, ${ }^{2}$ Gonçalo Nunes, ${ }^{2}$ Joana Bettencourt, ${ }^{3}$ Samuel Ângelo, ${ }^{4}$ Sónia Coelho, ${ }^{2}$ Vasco Maria ${ }^{5}$

\section{RESUMO}

Objectivos: Averiguar o impacte do aumento das taxas moderadoras na procura dos cuidados de saúde primários na USF do Parque. Comparar as afluências às consultas na USF do Parque nos períodos homólogos de 1 de Janeiro a 31 de Maio dos anos 2011 e 2012. Identificar outros factores relevantes na afluência dos utentes às consultas da USF.

Tipo de Estudo: Longitudinal retrospectivo, Observacional e Analítico.

Local: USF do Parque, ACES Lisboa Norte.

População: Utentes utilizadores da USF do Parque.

Métodos: Amostragem por conveniência. Colheita de dados realizada através de questionário e consulta dos registos informáticos da USF. Análise dos resultados através do programa SPSS Statistics versão 19, com nível de significância estatística de $5 \%$, com recurso aos testes $t$ de Student, Anova, correlação de Pearson e correlação de Spearman.

Resultados: Foram analisados 338 doentes com predomínio do sexo feminino ( $\mathrm{n}=241 ; 71,3 \%$ ), com uma média de idades de 57 anos (DP = 18,92). Registou-se um aumento do número de consultas de 2011 para 2012 de 0,87 consultas por utente (IC95\%: $0,623-1,129)(p<0,01)$. Utentes mais idosos, com mais patologias e medicação diária apresentam maior afluência às consultas $(p<0,05)$. Houve menos consultas em utentes cujo rendimento mensal do agregado é elevado $(p<0,05)$. Verificou-se ainda uma correlação positiva superior entre o número total de patologias e consultas na população isenta, em 2012 ( $p<0,05)$. Conclusões: O número de consultas em 2012 foi superior a 2011. A idade avançada, a multipatologia e a medicação diária justificam um maior número de consultas de seguimento. Um maior rendimento económico contribuiu para uma menor afluência em ambos os períodos. O aumento das taxas moderadoras não apresentou um impacte negativo no recurso às consultas da USF do Parque.

Palavras-Chave: Taxas Moderadoras; Acessibilidade; Cuidados de Saúde Primários.

\section{INTRODUÇÃO}

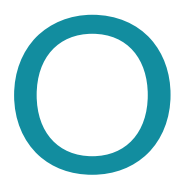

acesso a cuidados de saúde constitui um dos pilares fundamentais das sociedades modernas e revela-se essencial na promoção do bem-estar dos cidadãos mediante a prevenção, diagnóstico e terapêutica de múltiplas pa- tologias. Assim, circunstâncias económicas, sociais, organizacionais ou culturais não deveriam constituir barreiras efectivas a este processo. ${ }^{1}$

Os cuidados de saúde primários são o primeiro nível de contacto dos doentes com o sistema de saúde, desempenhando um papel central como orientadores do

${ }^{1}$ Licenciado em Medicina Veterinária pela Universidade de Évora. Mestre em Patologia Experimental pela Faculdade de Medicina da Universidade de Coimbra. Aluno do $6^{\circ}$ ano do Mestrado Integrado em Medicina na Faculdade de Medicina da Universidade de Lisboa.

${ }^{2}$ Aluno do $6^{\circ}$ ano do Mestrado Integrado em Medicina na Faculdade de Medicina da Universidade de Lisboa.

${ }^{3}$ Aluno do $5^{\circ}$ ano do Mestrado Integrado em Medicina na Faculdade de Medicina da Universidade de Lisboa.

${ }^{4}$ Licenciado em Medicina Dentária pela Faculdade de Medicina Dentária da Universidade de Lisboa. Aluno do $6^{\circ}$ ano do Mestrado Integrado em Medicina na Faculdade de Medicina da Universidade de Lisboa.

${ }^{5}$ Professor Auxiliar da Faculdade de Medicina da Universidade de Lisboa, Instituto de Medicina Preventiva. Regente da Disciplina de Medicina Geral e Familiar. 
doente dentro desta rede complexa e abrangente de cuidados médicos. A evolução mais significativa dos últimos anos, na reforma dos cuidados de saúde primários em Portugal, traduziu-se na criação de Agrupamentos de Centros de Saúde (ACES) e Unidades de Saúde Familiar (USF). Esta alteração tem como objectivo aproximar dos cidadãos a estrutura de prestação de cuidados de saúde, ao mesmo tempo que introduz uma flexibilização de organização e gestão face à estrutura de prestação de cuidados de saúde primários até então existente. $^{2}$

O Serviço Nacional de Saúde (SNS) vigente em Portugal, desde o seu estabelecimento e expansão, tem-se pautado por princípios fundamentais, destacando-se o seu carácter universal e tendencialmente gratuito. A par dos inúmeros avanços científicos, médicos e tecnológicos que podem contribuir para a melhoria da qualidade dos serviços, surgem questões relativas à eficiência e sustentabilidade do SNS, sobretudo no contexto actual de crise económica e financeira, em que se verifica uma escassez significativa de recursos. ${ }^{3}$

As taxas moderadoras constituem um instrumento financeiro, cujo objectivo primário consiste em racionalizar a procura de cuidados médicos. $\mathrm{O}$ aparecimento de novas taxas de internamento e cirurgia de ambulatório, bem como os recentes incrementos que têm sofrido, podem colocar em causa o princípio da equidade económica e social do acesso ao SNS ${ }^{4}$. A última actualização do valor das taxas moderadoras (Portaria n. ${ }^{\circ}$ 306-A/2011, de 20 de Dezembro) ${ }^{5}$ entrou em vigor a 1 de Janeiro de 2012, registando-se um aumento de 2,25€ para $5 €$ nas consultas de Medicina Geral e Familiar, e de $9,60 €$ para $20 €$ no atendimento em Serviço de Urgência Polivalente. ${ }^{6}$

Neste contexto, torna-se pertinente uma reavaliação dos factores que influenciam o acesso dos doentes aos cuidados de saúde, podendo o montante das taxas moderadoras tornar-se um parâmetro potencialmente impeditivo desse acesso, nomeadamente ao nível dos cuidados de saúde primários e sobretudo em indivíduos com baixo nível socio-económico.

Assim, o presente trabalho propõe averiguar o impacte do aumento das taxas moderadoras na procura dos cuidados de saúde primários na Unidade de Saúde Familiar (USF) do Parque, integrada no ACES Lisboa Norte. Como objectivos secundários pretende-se com- parar as afluências às consultas na USF nos períodos homólogos de 1 de Janeiro a 31 de Maio dos anos 2011 e 2012, bem como identificar outros factores relevantes na afluência dos utentes às mesmas.

\section{MÉTODOS}

Realizou-se um estudo do tipo longitudinal retrospectivo, observacional e analítico. A população alvo foi constituída pelos utentes utilizadores da USF do Parque. Recorreu-se a uma amostragem de conveniência, constituída pelos utentes que se dirigiram à USF no período compreendido entre 22 e 31 de Maio de 2012. A dimensão inicial da amostra foi calculada em 384 utentes, com recurso à fórmula $n=p(1-p) /(E / 1.96)^{2}$ (sendo $n$ a dimensão mínima da amostra, $p$ a máxima prevalência esperada da principal variável dependente (afluência à USF) - 50\% e $E$ o erro de precisão da estimativa -5\%).

As variáveis utilizadas para o estudo, avaliadas por resposta a um questionário foram: idade; sexo; habilitações literárias; número de elementos do agregado familiar; rendimento médio mensal do agregado familiar; acessibilidade - variável composta avaliada pela distância média da residência à USF, meio de transporte utilizado na deslocação à USF (a pé; autocarro; metro; táxi; viatura própria) e capacidade de locomoção (sem auxiliares de marcha; recurso a auxiliares de marcha; cadeira de rodas; alectuação); isenção actual de taxa moderadora (gravidez; doença; insuficiência económica; outro motivo não especificado; sem isenção); relação médico-doente (muito boa; boa; satisfatória; insatisfatória; má); doenças crónicas (hipertensão arterial; diabetes mellitus; dislipidémia; depressão; doença reumática; cancro; outras não especificadas) e número de medicamentos tomados diariamente. As variáveis dependentes foram: número de consultas no período de 1 de Janeiro a 31 de Maio de 2011 e número de consultas no período de 1 de Janeiro a 31 de Maio de 2012.

O questionário foi aplicado aos utentes de ambos os sexos e com idade igual ou superior a 16 anos que recorreram às consultas de Saúde do Adulto, Planeamento Familiar, Saúde Materna, bem como a consultas de urgência durante o período do estudo. Definiu-se como único critério de exclusão do estudo a não indicação do número de beneficiário do SNS (face à impossibilidade inerente de acesso aos dados do siste- 
ma informático da USF). Todavia, os utentes que, apesar de terem preenchido o questionário, não responderam à totalidade das questões, foram posteriormente eliminados da análise dos resultados. Mediante a consulta dos respectivos processos clínicos informatizados, compararam-se as afluências às consultas médicas nos períodos homólogos de 1 de Janeiro a 31 de Maio dos anos de 2011 e 2012. Todos os inquiridos deram o seu consentimento informado para a participação no estudo. Foi ainda solicitada e obtida autorização do coordenador da USF para a realização do estudo e redigida uma declaração, onde todos os elementos do grupo de investigação se comprometeram a manter a confidencialidade dos dados obtidos.

Para a presente análise de resultados recorreu-se ao programa SPSS Statistics versão 19, utilizando-se um nível de significância estatística de $5 \%(p<0,05)$. Realizouse uma análise descritiva dos resultados obtidos em termos de frequências e percentagens, obtendo uma visão geral dos mesmos. Recorreu-se também a testes de estatística inferencial, de modo a analisar a significância das relações existentes entre as variáveis em estudo e o número de consultas em 2011 e 2012. Para tal foi analisada a normalidade da distribuição dos resultados obtidos ao nível dos grupos com número mais reduzido de elementos, tendo em consideração os pressupostos do teorema do limite central. Uma vez que esses resultados apresentam uma distribuição normal, recorreu-se a testes paramétricos para analisar a sua significância estatística. Assim, de forma a comparar as diferenças entre o número de consultas de 2011 e 2012, recorreu-se ao teste $t$ de Student para amostras emparelhadas e independentes. Para comparar mais que 2 grupos utilizou-se o teste Anova com o respectivo teste LSD (Least Significant Difference) para posterior comparação múltipla de médias. Utilizou-se o teste de correlação de Spearman (não paramétrico) para verificar a correlação entre variáveis ordinais e intervalares e o teste de correlação de Pearson para variáveis quantitativas.

\section{RESULTADOS}

Foram analisados dados relativos a 338 utentes que recorreram à consulta durante o período do estudo (após anulação de 46 por se verificarem os critérios de exclusão) com uma média de idades de 57 anos $(D P=$ 18,92) e com um mínimo de 16 e um máximo de 91 anos.
A maioria dos elementos é do sexo feminino ( $n=241$; $71,3 \%)$. Um número maior de participantes possui o ensino superior $(n=91 ; 26,9 \%)$, seguindo-se os que tem apenas o $1 .^{\circ}$ ciclo $(n=84,24,9 \%)$ e os que tem o ensino secundário ( $n=76 ; 22,5 \%)$. Um número mais reduzido tem apenas o $3 .{ }^{\circ}$ ciclo $(n=40 ; 11,8 \%)$, o $2 .^{\circ}$ ciclo $(n=37$, $10,9 \%)$ ou são analfabetos ( $n=10 ; 3 \%)$. A maioria dos inquiridos pertence a um agregado familiar constituído por dois elementos ( $n=98 ; 29 \%$ ).

Em relação ao rendimento mensal auferido pelo agregado, a maior parte dos utentes afirma receber entre 500 a 1000 euros $(n=110 ; 32,5 \%)$. Um número inferior recebe entre 2000 a 2500 euros mensais $(n=24$; $7,1 \%)$.

No que se refere à distância média percorrida pelos utentes da residência até à USF, os resultados indicam que a maioria ( $n=273 ; 80,8 \%$ ) percorre menos de $5 \mathrm{~km}$, seguindo-se em menor número os que percorrem entre 5 a $15 \mathrm{~km}(n=51 ; 15,1 \%)$, entre 15 a $30 \mathrm{~km}(n=10$; $3 \%)$ e aqueles que se deslocam mais de $30 \mathrm{~km}(n=4$; $1,2 \%)$. Quanto ao meio de transporte utilizado pelos utentes na deslocação à respectiva USF, os resultados revelam que a maioria ou vai a pé $(n=122 ; 36,1 \%)$ ou desloca-se em viatura própria $(n=120 ; 35,5 \%)$. Um número mais reduzido refere ir de autocarro $(n=74$; $21,9 \%)$, de metro ( $n=12 ; 3,6 \%)$ ou de táxi $(n=10 ; 3 \%)$. Em relação à capacidade de locomoção, os resultados apontam para um número superior de indivíduos que se encontram autónomos sem recurso a qualquer tipo de auxiliares de marcha $(\mathrm{n}=314 ; 92,9 \%)$. Um número bem mais reduzido recorre a auxiliares de marcha $(n=$ $22 ; 6,5 \%)$, anda em cadeira de rodas ( $n=1 ; 0,3 \%$ ) ou não tem capacidade de locomoção $(n=1 ; 0,3 \%)$.

Relativamente ao modo como os indivíduos avaliam a dificuldade de acesso à USF em termos de distância, meio de transporte e facilidade de locomoção, constata-se que a maior parte considera a acessibilidade fácil ( $n=156 ; 46,2 \%$ ), seguindo-se os que a consideram muito fácil ( $n=103 ; 30,5 \%)$, razoável $(n=53 ; 15,7 \%)$, difícil ( $n=24 ; 7,1 \%)$ e muito difícil ( $n=2 ; 0,6 \%$ ).

A análise do tipo de isenção das taxas moderadoras, observada na figura 1 , indica que um número superior de participantes não estão isentos ( $n=213$; $63 \%)$, seguindo-se em número consideravelmente inferior os isentos por insuficiência económica $(n=65 ; 19,2 \%)$, por doença $(n=41 ; 12,1 \%)$, por outro motivo não es- 
pecificado $(n=10 ; 3 \%)$ ou por gravidez $(n=9 ; 2,7 \%)$.

$\mathrm{Na}$ análise da relação médico-doente, os resultados apontam para um número elevado de inquiridos que consideram a mesma muito boa $(n=172 ; 50,9 \%)$. Em número mais reduzido encontram-se os que a consideram boa ( $n=124 ; 36,7 \%)$, satisfatória $(n=35 ; 10,4 \%)$ e insatisfatória $(n=7 ; 2,1 \%)$.

A análise da figura 2 mostra que a maior parte dos utentes sofrem de hipertensão arterial (37,9\%), seguindo-se os que tem doenças reumáticas $(30,2 \%)$, dislipidémia $(29,9 \%)$, depressão $(16,3 \%)$, diabetes mellitus $(11,8 \%)$ e cancro, estes últimos em percentagem mais reduzida $(8 \%)$.

Da observação dos resultados referentes à medicação diária (quadro I), podemos concluir que cerca de um terço dos inquiridos $(n=105 ; 31,1 \%)$ não consomem nenhum medicamento.

Nos elementos da amostra em estudo, verificou-se um total de 672 consultas no período referente ao ano 2011: $\mathrm{M}=1,99$; (IC95\%: 1,76-2,22) e 968 consultas em período homólogo de 2012: $\mathrm{M}=2,86$; (IC95\%: 2,623,11 ). Os resultados do teste t de Student para amostras emparelhadas revelaram existir diferenças estatisticamente significativas entre o número de consultas realizadas em 2011 e em 2012: M = 0,87 (IC95\%: 0,623$1,129)(p<0,05)$.

Em relação à idade, o teste de correlação de Spearman revela uma correlação positiva e estatisticamente significativa entre a idade dos pacientes inquiridos e o número de consultas, quer em $2011(\mathrm{r}=0,23$; $p<0,01)$, quer em $2012(\mathrm{r}=0,28 ; p<0,01)$, o que significa que a idades mais avançadas correspondem também um maior número de consultas. Contudo, a idade não se revelou um factor significativo quando comparada com a diferença em relação ao número de consultas nos dois períodos ( $p$ $\geq 0,05$ ).

Verificou-se um menor número de consultas nos indivíduos com maior rendimento médio mensal, quer em 2011 quer em $2012(p<0,05)$, contudo quanto à diferença entre o número de consultas nos dois períodos, este factor não se revelou estatisticamente significativo $(p \geq 0,05)$.

Nas variáveis relativas à acessibilidade

\begin{tabular}{|c|c|c|}
\hline \multicolumn{3}{|c|}{$\begin{array}{l}\text { QUADRO I. Distribuição da medicação diária } \\
\text { consumida pelos elementos da amostra. }\end{array}$} \\
\hline $\begin{array}{l}\text { Número de medicamentos } \\
\text { consumidos diariamente }\end{array}$ & $\mathbf{n}$ & Percentagem \\
\hline Nenhum medicamento & 105 & 31,1 \\
\hline 1 Medicamento & 44 & 13,0 \\
\hline 2 Medicamentos & 49 & 14,5 \\
\hline 3 Medicamentos & 36 & 10,7 \\
\hline 4 Medicamentos & 29 & 8,6 \\
\hline 5 Medicamentos & 17 & 5,0 \\
\hline$\geq 6$ Medicamentos & 58 & 17,2 \\
\hline
\end{tabular}

(distância média da residência à USF, meio de transporte utilizado, capacidade de locomoção) não se verificaram diferenças estatisticamente significativas na variação do número de consultas nos dois períodos em estudo ( $p \geq 0,05$ ). O tipo de isenção (quadro 2 ), a que os utentes inquiridos estão sujeitos também está relacionado com o número de consultas na USF, tanto no ano de 2011 como no ano de $2012(p<0,05)$, registando-se um número superior de consultas nos isentos por insuficiência económica, por doença e por gravidez ( $p<$ $0,05)$. Contudo as diferenças observadas entre $2012 \mathrm{e}$ 2011 não podem ser atribuídas ao tipo de isenção ( $p \geq$ $0,05)$, sendo os valores semelhantes nos diversos grupos.

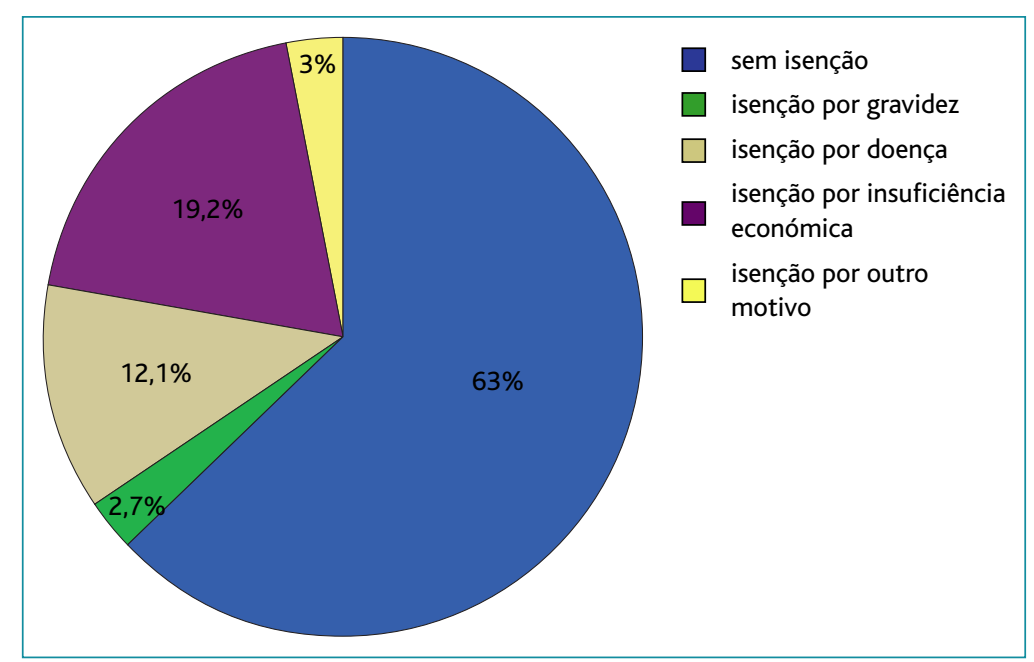

Figura 1. Isenção de taxa moderadora. 


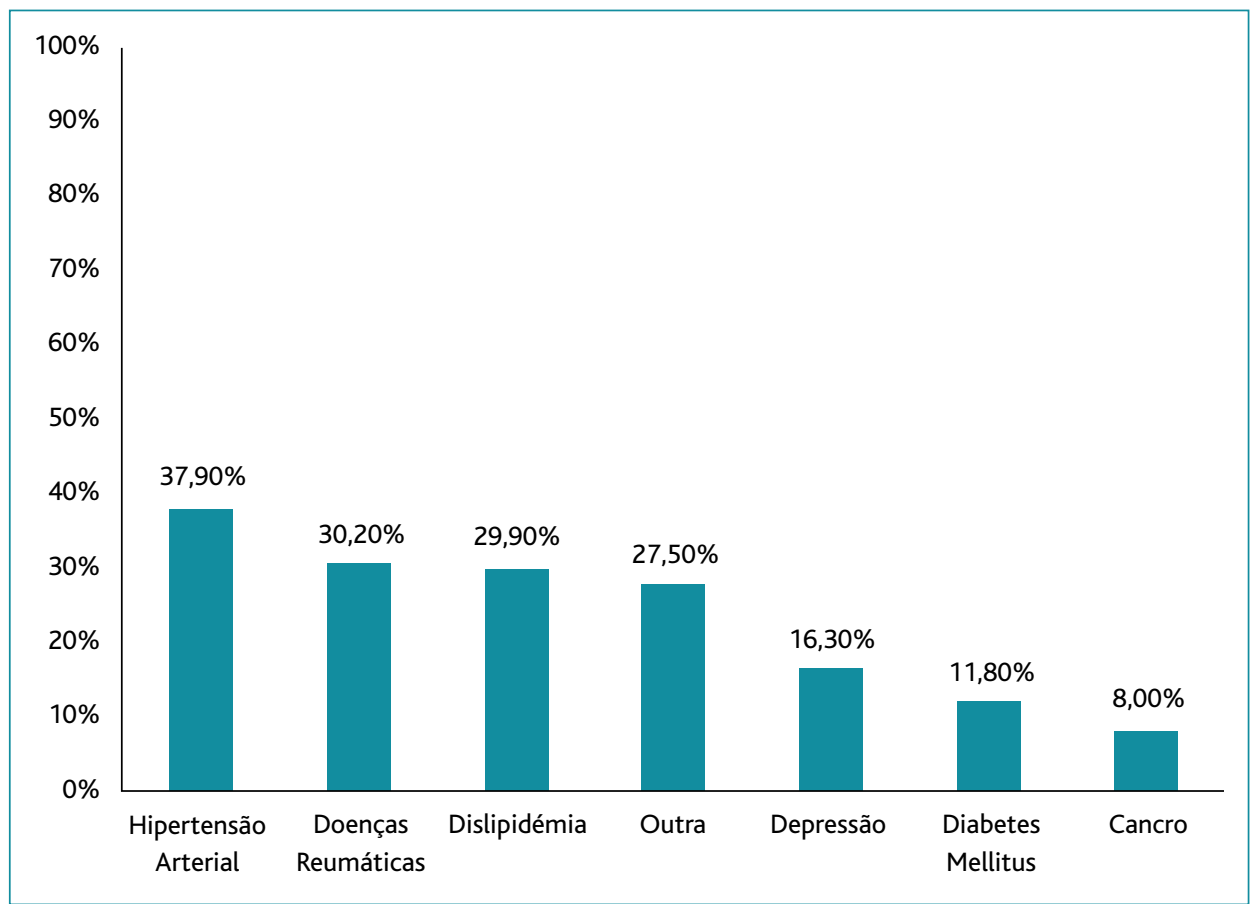

Figura 2. Doenças Crónicas.

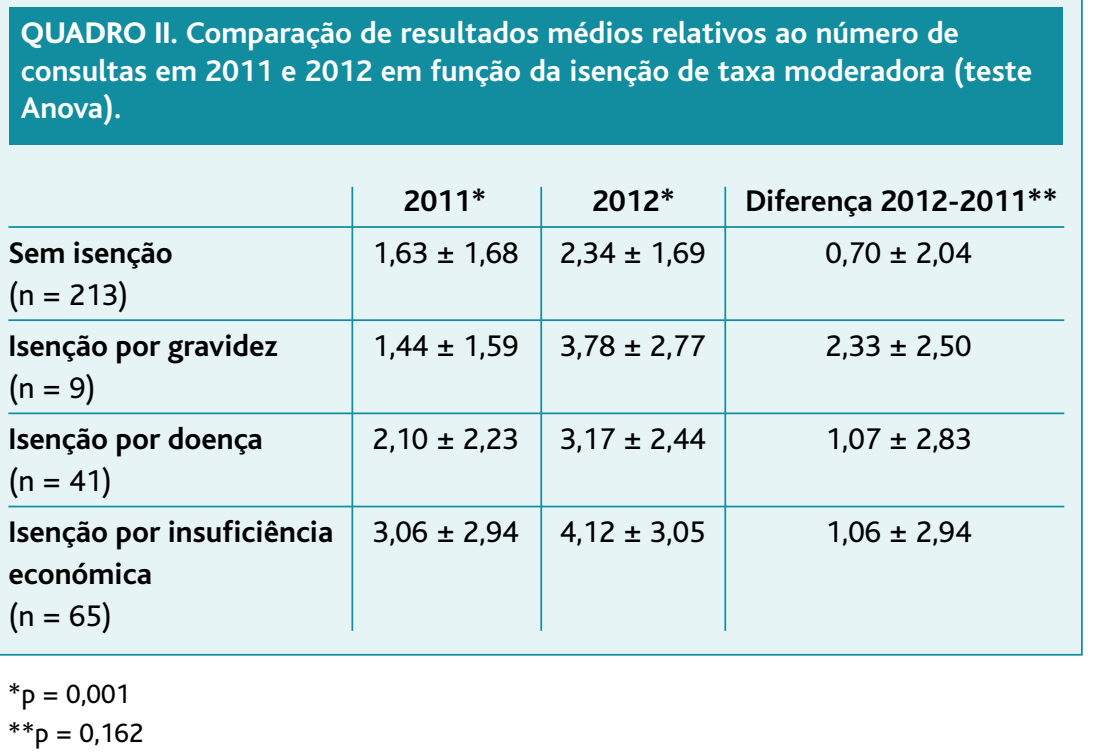

lação de Pearson constatouse que o número total de patologias por utente correlaciona-se positivamente com o número de consultas em 2011 e $2012(p<0,01)$, sem que a diferença entre os dois períodos tivesse sido estatisticamente significativa $(p \geq 0,05)$.

A relação entre o número de medicamentos consumidos diariamente e o número de consultas apresentou-se positiva e estatisticamente significativa em 2011 ( $r=0,29 ; p<0,01)$ e 2012 ( $r=0,405 ; p<0,01)$, assim como no que se refere à diferença verificada entre os dois anos $(\mathrm{r}=$ 0,405; $p<0,05$ ).

Podemos ainda constatar pelo teste de Correlação de Pearson, cujos resultados estão patentes no quadro 4, que existe uma correlação positiva entre o número total de patologias e o número de consultas, tendo esta sido mais forte no ano de 2011 nos utentes não isentos, e em 2012 nos isentos do pagamento de taxas moderadoras $(p<0,05)$.

As variáveis anteriores foram ainda correlacionadas nos utentes em que o rendimento do agregado é inferior a 1000 euros, verificando-se uma correlação positiva e significativa em ambos os anos no grupo não isento $(p<0,05)$, e apenas em 2012 no grupo de isentos $(p<0,01)$.
A análise comparativa do número de consultas, face a existência ou não de qualquer tipo de doença, não revela diferenças estatisticamente significativas nos dois anos em estudo, de acordo com o teste t de Student para amostras independentes $(p \geq 0,05)$. Todavia, no teste de Corre-

\section{DISCUSSÃO}

A introdução de taxas moderadoras tem como finalidade racionalizar a procura de cuidados de saúde. Assim sendo, após o recente aumento do montante das referidas taxas, seria de esperar um decréscimo na 
QUADRO III. Correlação de Pearson entre o número de patologias e o número de consultas em 2011 e 2012 nos grupos de isentos e não isentos (indivíduos com agregado cujo rendimento é inferior a 1000€).

\begin{tabular}{|l|c|c|c}
\multicolumn{2}{l|}{ Isenção de taxa moderadora } & 2011 & 2012 \\
\hline Não & $\begin{array}{c}\text { Número de patologias } \\
(\mathrm{n}=95)\end{array}$ & $0,327^{*}$ & $0,222^{*}$ \\
\hline Sim & $\begin{array}{c}\text { Número de patologias } \\
(\mathrm{n}=84)\end{array}$ & 0,200 & $0,389^{*}$ \\
\hline
\end{tabular}

${ }^{*} \mathrm{p}<0,01$

afluência às consultas na USF do Parque, o que não se verificou, de acordo com os resultados obtidos neste estudo. De acordo com dados estatísticos disponibilizados pela USF do Parque, entre 1 de Janeiro e 31 de Maio de 2011, realizaram-se nesta unidade 4827 consultas de adultos, e no período homólogo de 2012, 5571 consultas. Esta realidade, que corrobora os resultados da amostra estudada, pode ser justificada pelo facto da USF do Parque ser uma instituição recente, inaugurada no ano de 2009, com a atribuição de médicos de família a todos os inscritos. Por outro lado, uma atitude pró-activa da unidade na divulgação de informação sobre os diferentes tipos de serviços disponibilizados pode ter contribuído para uma melhoria na acessibilidade e consequentemente para uma maior afluência.

Os utentes que se deslocaram com maior frequência, por motivo de consulta, à USF nos períodos em estudo são os mais idosos, e aqueles que tomam maior número de medicamentos, tal como se pode observar pelos resultados estatisticamente significativos que se observaram nas variáveis «idade», «doenças crónicas» $\mathrm{e}$ «medicação diária». Estes dados poderão ser explicados pelo facto do aumento da idade ser um factor predisponente para os indivíduos apresentarem multipatologia que justifique o recurso a vários fármacos e deste modo, ser necessário um maior número de consultas de seguimento e com intervalos mais curtos. Os elementos da amostra pertencentes a agregados familiares de rendimento mais elevado recorreram com menor frequência às consultas nos anos 2011 e 2012, o que se pode justificar pela já conhecida existência de uma relação positiva entre o nível socio-económico e a saúde individual. ${ }^{7}$ Adicionalmente, é possível que indiví- duos com capacidade económica superior possam recorrer com maior facilidade a serviços de saúde privados, e deste modo com menor frequência ao seu médico de família, aspecto que poderia explicar até certo ponto os resultados obtidos. Comparando os utentes isentos e não isentos de pagamento de taxas moderadoras, com número de patologias idêntico, verifica-se um comportamento similar no ano de 2011, sendo que em 2012, essa correlação positiva apenas se observa na população isenta. Este achado faz-nos supor que estes utentes, dada a ausência de taxação, não sentiram qualquer impacte económico directo em relação aos custos inerentes aos cuidados de saúde prestados, que justificasse uma diminuição da afluência.

Na realização deste estudo, a utilização de uma amostra de conveniência constituiu uma limitação relevante na extrapolação dos resultados obtidos para a população alvo. Por outro lado, todo o estudo foi baseado no pressuposto de que a única variável, alvo de análise estatística inferencial, que sofreu alteração de 2011 para 2012 foi o valor da taxa moderadora. Adicionalmente, não foi levada em consideração a recente alteração das normas de atribuição de isenção que entretanto se operou no SNS, podendo ter-se introduzido um viés de confundimento. Reconhece-se ainda que o apuramento do rendimento económico, apenas com base no rendimento médio mensal e dimensão do agregado familiar, pode ser redutor e insuficiente para a caracterização do mesmo.

O presente estudo demonstra que o número de patologias crónicas e o número de medicamentos diários constituem os principais determinantes na afluência às consultas na USF do Parque. Apesar da ausência de diferenças estatisticamente significativas entre os dois períodos homólogos em estudo, regista-se uma maior correlação dos factores supracitados com o número de consultas realizadas entre Janeiro e Maio de 2012 na população isenta.

Em suma, o aumento das taxas moderadoras não teve um impacte negativo directo no recurso às consultas na USF do Parque, com base na contagem total de consultas efectuadas pelos elementos da amostra. Para tal facto, pode ter contribuído o aumento ainda mais preponderante das taxas moderadoras nos serviços de urgência hospitalares e a atitude pró-activa de uma USF constituída recentemente. $\mathrm{O}$ achado de que indivíduos isentos apresentam uma afluência superior 
comparativamente a indivíduos não isentos com idêntico número de patologias sugere que estudos adicionais deverão ser efectuados, por períodos de tempo mais longos e recorrendo a amostras aleatorizadas e de maior dimensão, de forma a aferir o real impacte do aumento das taxas moderadoras no acesso aos cuidados de saúde primários.

\section{AGRADECIMENTOS}

Os autores agradecem ao Professor Doutor Luís Rebelo, coordenador da USF do Parque, a disponibilidade demonstrada, essencial na recolha dos dados necessários à elaboração do presente trabalho.

\section{REFERÊNCIAS BIBLIOGRÁFICAS}

1. Furtado C, Pereira J. Equidade e Acesso aos Cuidados de Saúde: documento de trabalho. Lisboa: Escola Nacional de Saúde Pública da Universidade Nova de Lisboa; 2010.

2. Barros PP. Economia da Saúde - conceitos e comportamentos. $2^{\mathrm{a}}$ ed. rev. Coimbra: Almedina; 2012.

3. Miguel JP, Bugalho M. Economia da saúde: novos modelos. Análise Social 2002; 38 (166): 51-75.

4. Barbosa AP. Implicações éticas das taxas moderadoras face à escassez de recursos em saúde [dissertação de mestrado]. Porto: Faculdade de
Medicina da Universidade do Porto; 2009.

5. Ministérios das Finanças e da Saúde. Portaria n 306-A/2011, de 20 de Dezembro. Diário da República n 242/2011 - I Série.

6. Administração Regional de Saúde. Circular Normativa $n^{\circ}$ 3/2006, de 14 de Agosto. Devolução de Taxas Moderadoras - Porto: ARS Norte; 2006.

7. Oliveira DG. Determinantes do estado de saúde dos portugueses [dissertação de mestrado]. Lisboa: Instituto Superior de Estatística e Gestão de Informação da Universidade Nova de Lisboa; 2010.

\section{CONFLITOS DE INTERESSE}

Os autores declaram não possuir conflitos de interesse.

\section{FINANCIAMENTO}

O trabalho não foi objecto de qualquer tipo de financiamento externo (incluindo bolsas de investigação).

\section{ENDEREÇO PARA CORRESPONDÊNCIA}

Gonçalo Filipe Domingos Nunes

Alameda Salgueiro Maia, № $159^{\circ} \mathrm{B}$ - Flamenga;

2660-329 - Santo António dos Cavaleiros

gonçalo.n@hotmail.com

Recebido em 26/09/2012

Aceite para publicação em 26/03/2013

\section{ABSTRACT}

\section{THE IMPACT OF THE INCREASE IN USER FEES ON THE DEMAND FOR PRIMARY HEALTH CARE IN THE PARQUE FAMILY HEALTH UNIT}

Objectives: To assess the impact of the increase in user fees on the demand for primary health care in the Parque Family Health Unit, to compare consultation rates in the Parque FHU between January 1and May 31, 2011, and the same period in 2012, and to identify factors associated with patient demand for care in this unit.

Design: Retrospective longitudinal, observational and analytical.

Setting: Parque Family Health Unit, North Lisbon Health Centres Group (ACES Lisboa Norte) Population: Patients of the Parque Family Health Unit.

Methods: A convenience sample of patients was selected from the health records of the Family Health Unit. The Student's $\mathrm{t}$-test, Anova, Pearson correlation and Spearman correlation were used with statistical significance set at the $5 \%$ level.

Results: We analyzed the records of 338 patients. The majority were female $(n=241 ; 71,3 \%)$, with a mean age of 57 years (standard deviation $=18,92$ ). There was an increase in the consultation rate from 2011 to 2012 . The mean number of visits was 0.87 (IC95\% 0.623-1.129, $p<0.01$ ). Older patients, patients with more illnesses, and those taking daily medication had higher visiting rates $(p<0.05)$. There were fewer visits made by patients with a higher monthly income $(p<0.05)$. A positive correlation between the number of illnesses and the number of medical visits was observed in patients exempt from user fees.

Conclusions: In 2012 the number of visits to the Health Unit increased. Advanced age, a larger number of illnesses and daily medication use were associated with a greater number of visits. Higher economic status was associated with lower visiting rates in both study periods. The increase in user fees did not have a negative impact on patient demand for primary health care in the Parque Family Health Unit.

Key Words: User Fees; Accessibility; Primary Health Care. 